\title{
El Riesgo como un Obstáculo \\ para la Equidad en la Salud
}

\author{
Risk as an Obstacle for Health Equity
}

Carla Flores Figueroa ${ }^{1}$

\begin{abstract}
Resumen
El objetivo de este artículo es defender la tesis de que el uso indiscriminado del concepto "riesgo" en el sistema de salud termina limitando las posibilidades de transferir derechos a la ciudadanía y al mismo tiempo avanzar hacia la equidad.
\end{abstract}

En primer lugar, para probar esto, se presentan argumentos que demuestran que los procesos de definición de lo que es riesgoso o no, responden a cuestiones ideológicas y no a cuestiones de naturaleza necesariamente científica, ni medible.

En segundo lugar, se demuestra que los programas responsables de definir y evaluar el riesgo enfatizan en la definición y valoración de los déficits individuales, ignorando las barreras estructurales que existen en el país. Además, asocian el riesgo a peligro, cuestión que provoca que se discrimine todo lo que se considere en esta categoría.

Por último, se propone utilizar las perspectivas teóricas que también observen las fuerzas y el capital social que poseen las personas y las comunidades, con el objeto de dejar de ver los indicadores de riesgo como cuestiones naturales y fácticas y confiar en todos las personas y categorías que son consideradas peligrosas.

Palabras Clave: Riesgo, peligro, ciudadanía, equidad en salud

\begin{abstract}
The objective of this article is to defend the thesis that the indiscriminate use of the concept "risk" in the health system ends up limiting the possibilities of transferring rights to citizens and at the same time moving towards equity.

First, to prove this, arguments are presented that demonstrate that the processes of defining what is risky or not, respond to ideological questions and not to questions of a necessarily scientific or measurable nature.

Secondly, it is shown that the programs responsible for defining and assessing risk emphasize the definition and assessment of individual deficits, ignoring the structural barriers that exist in the country. In addition, they associate the risk with danger, an issue that causes everything that was consider in this category to be discriminated against.

Finally, it is proposed to use the theoretical perspectives that the forces and social capital possessed by people and communities also observe, in order to stop seeing risk indicators as natural and factual issues and trust all people and categories. They are considered dangerous.
\end{abstract}

Key words: Risk, danger, citizenship, health equity

1. Doctora $\odot$ en Salud Pública de la Universidad de Chile, Magíster en Trabajo Social de la Pontificia Universidad Católica. Adscripción Institucional: Coordinadora de Pregrado de la Faculta de Salud, Universidad Santo Tomás. Dirección: Avenida Ejército \#146, Santiago, Chile. Teléfono Celular: +56983548584 Correo electrónico: carlaflores@santotomas.cl 


\section{Contextualización}

Durante la primera mitad del siglo pasado hubo un gran desarrollo de la epidemiología y de los métodos estadísticos para estudiar las causas y ocurrencias de las enfermedades. No obstante, la disminución de las enfermedades transmisibles y el aumento de las crónicas provocaron que a principios de 1970 surgieran fuertes críticas respecto al excesivo énfasis que ponían los epidemiólogos en el estudio de su sustrato biológico.

Es este el escenario en el que surge el interés de los salubristas por demostrar cómo los determinantes sociales influyen y delimitan la salud de las poblaciones. De esto se ocuparía la epidemiología social, la que trata de "investigar explícitamente los determinantes sociales de las distribuciones de la salud, la enfermedad y el bienestar en las poblaciones, en vez de tratar dichos determinantes como un simple trasfondo de los fenómenos biomédicos" (Krieger, 2002).

A la fecha se han elaborado más de 15 modelos conceptuales que tratan de explicar cómo estos factores provocan determinados resultados de salud. Sin embargo, únicamente Irwin y Solar, en el marco de la Comisión de Determinantes Sociales de la Organización Mundial de la Salud, han integrado el sistema de salud como uno de los mecanismos que genera gradientes de estratificación social que afectan la calidad de vida de las personas de forma diferenciada.

Para estos autores, el sistema de salud se convierte en un elemento particularmente importante a la hora de pensar en reducir el impacto de las desigualdades sociales. No solo porque es un medio para asegurar el acceso equitativo a la atención, sino porque además a través de él se pueden realizar acciones de promoción y prevención que permitan mejorar la calidad de vida de las poblaciones más vulnerables.

Ahora bien, no es posible pensar en todas las oportunidades que ofrece el sistema de salud para disminuir las inequidades, sin reflexionar sobre las características de las estructuras que lo conforman. Sin ir más lejos, durante la última década se ha tratado de instalar el enfoque de derechos en el sistema de salud chileno, pero las relaciones de poder, la cultura, las prácticas y los valores de quienes integran los equipos que deben implementar estos cambios, se han transformado en verdaderos obstaculizadores de esta reforma (Programa de Naciones Unidas para

el Desarrollo, 2009).

Esto ocurre, porque este tipo de reformas, por una parte, busca generar un sistema de atención en que los usuarios sean abordados como ciudadanos, autónomos, capaces y responsables de su salud, pero, por otra parte, se espera esto dentro de espacios institucionales y organizacionales que se han caracterizado durante años por vigilar y controlar a aquellos "que se cree son incapaces de administrar sus propias vidas" (Beddoe, 2010) a los sujetos que se califican como "en riesgo".

Es por ese motivo, que el presente artículo busca analizar cómo el uso desmedido del concepto de riesgo se ha transformado en un mecanismo a través del cual el sistema de salud termina limitando las posibilidades de transferir derechos a la ciudadanía y al mismo tiempo avanzar hacia la equidad.

2. Con anterioridad Diderichsen, Benzeval, Judge y Whitehead habían hablado del sistema de salud como un elemento para enfrentar las inequidades, pero no lo habían definido como un determinante social de la salud. 


\section{La definición del riesgo, una cuestión ideológica no científica}

Los avances en epidemiología y en estadística sanitaria, han evolucionado junto al desarrollo de software (SPPS, STATA y R) que permiten procesar inimaginables cantidades de información. Esto es importante porque a partir de ellos es posible describir detalladamente los factores de riesgo que aquejan a las poblaciones más vulnerables, perpetuando así el esquema de autoridad propio de las ciencias biomédicas.

No obstante, como se puede observar en los siguientes titulares de prensa de circulación nacional, la consideración del riesgo en salud no es de exclusivo interés de la ciencia:

"Informe del MINSAL revela alza de extranjeros notificados por VIH/Sida. "Son múltiples los factores socioculturales, económicos y políticos, tanto en los países de origen como de destino, que inciden en el riesgo y vulnerabilidad frente al VIH de las personas migrantes: normas sociales y culturales, barreras lingüísticas, pobreza, que genera acceso insuficiente a la información relativa a la prevención y a los servicios de salud"(El Mercurio, 2019).

"Nivel socioeconómico de la niñez, etnia y alfabetización, pueden tener una mayor influencia en el riesgo de deterioro cognitivo al final de la vida" (La Tercera, 2018).

"Ministra de Salud reconoce que no se respondió oficio del Sename en caso de niña que murió tras no recibir trasplante. La secretaria de Estado señaló que se evaluó la pobreza de la menor como "un riesgo" además de que no se tenía la certeza de que alguien se haría cargo de ella después de la operación. Asimismo, el hecho de que viviría en Chiloé también incidió" (El Mercurio, 2019).

En este contexto, es posible apreciar que los indicadores de pobreza, de nivel educacional, de ingresos, de territorio y de raza no se observan, ni tratan de forma neutral, es decir, también son elementos que permiten general juicios negativos sobre la población más vulnerable del país.

Además, tal como ha sido factible de observar en el contexto del estallido social, el riesgo se ha convertido en una palabra de moda, tanto en el lenguaje cotidiano de los medios de comunicación, como en el tecnicismo de las políticas públicas. Es más, es posible afirmar que es el enemigo invisible de los gobiernos de turno, por este motivo, no es de extrañar que las autoridades emprendan cruzadas personales e institucionales para identificarlo y contrarrestarlo, tal como se puede apreciar en los siguientes relatos:

"MINSAL detalla seguimiento de casos "en riesgo" de coronavirus: se les recomienda evitar lugares concurridos. La subsecretaria Paula Daza precisó que no se trata todavía de "sospechosos" y que la red de salud está preparándose para enfrentar una posible emergencia” Subsecretaria de Salud Pública, Paula Daza (Mercurio, 2020)

"Con una nueva Carta Magna está en riesgo -en el ámbito educacional- la libertad de los padres a escoger entre un colegio en que a un niño lo hagan sacarse la ropa a los 4 años y tocar al compañero u otro en el que le enseñen una sexualidad de manera distinta" Gonzalo Carrera, Conductor de Radio Agricultura (El Ciudadano, 2020)

"Los hijos de madre soltera tienen más riesgo de tener menos coeficiente intelectual. El hijo de una familia de padres normales puede llegar a 200 puntos. En cambio, el de padres separados a 150 ó 160 y el hijo de madre soltera a veces alcanza 100" Miguel Otero- Ex Senador Renovación Nacional (El Mostrador, 2014)

Si bien el riesgo es fácil de llevar a datos numéricos, en especial porque "estamos en una sociedad que le otorga valor a todo lo que se puede medir" (Arellano, 2010), en los extractos de los discursos presentados, quienes utilizan la palabra riesgo parecen hacerlo de forma objetiva, aunque no responda necesariamente a una apreciación científica sobre los hechos. Al respecto, Ulrich Beck plantea que esto ocurre porque la definición de los riesgos siempre supone la lucha de visiones de diversos actores y grupos de intereses (Montenegro, 2005), por tanto, se trata de algo subjetivo e ideológico, y no de una cuestión neutral y numérica. 
A lo anterior se añade que "las sociedades contemporáneas se caracterizan por un aumento de la cuantificación de los fenómenos sociales a través del uso de indicadores y por una alta presencia de esos números en la esfera de debate público" (Montenegro, 2005) que no necesariamente responde a los complejos problemas que enfrentan las poblaciones más vulnerables. Por lo demás, la enunciación de los riesgos pasa por quien los define y no por quién es identificado como parte de esa categoría discursiva.

En resumen, la definición de los riesgos es una cuestión ideológica de las más problemáticas, porque detrás de su carácter supuestamente científico, neutral y medible está el peligro de asumir un enfoque centrado en las carencias y no en una real oferta de oportunidades a quienes más lo necesitan. Asimismo, lo más crucial y paradójico es que en Salud, toda la Institucionalidad Internacional y Nacional, es políticamente correcta a la hora de describir los factores que afectan la salud de las personas, pero no da cuenta de cómo las categorías (género, etnia, edad, etc.) que se establecen como riesgosas son portadoras y objeto de discriminación por sí mismas.

\section{Usuarios etiquetados "de riesgo"}

El sistema se salud se caracteriza por estar permanentemente preocupado por la detección, comprensión y vigilancia de los factores de riesgo que pueden afectar a una población determinada. Ejemplo de esto es el sistema de alerta temprana, Chile Crece Contigo, que tiene por finalidad detectar e intervenir tempranamente los factores que puedan producir inequidad en el desarrollo de las personas.

Esto último es importante, porque a través de la identificación y caracterización del riesgo se justifica la entrega de servicios focalizados como una forma de proteger a las poblaciones más vulnerables. Especialmente en países como Chile, caracterizados por poseer escasos recursos para la inversión pública lo que deviene en prestaciones focalizadas, sin poder responder a las necesidades de toda la población.

El problema estriba en que los programas responsables de definir y evaluar el riesgo enfatizan en la definición y valoración de los déficits individuales, ignorando las barreras estructurales que el propio sistema de salud les impone. Por ejemplo, el programa Elige Vivir Sano pretende que las personas gestionen su propio riesgo, a través del ejercicio y de la "elección de un estilo de vida" (Kemshall, 2010) (alimentación, actividad física, etc.) saludable. Desde esta perspectiva quienes accedan a asumir esta responsabilidad serán recompensados a través de su inclusión en la sociedad y quienes no lo hagan serán excluidos por tomar decisiones que son consideradas peligrosas (Kemshall, 2010).

Por otra parte, "aun cuando una aproximación centrada en el problema puede focalizarse en factores institucionales, la influencia del modelo biomédico alimenta la tendencia a enfocar el análisis de estos desde las deficiencias individuales" (Kenneddy, 1995). Por ejemplo, aunque los programas de promoción de salud apunten a que la comunidad ejerza un mayor control sobre los determinantes sociales para mejorar su calidad de vida, las intervenciones que de ellos se desprenden (consejería de salud, educación de adultos, etc.) tienden a centrarse en la conducta, como categoría de responsabilidad individual (abuso de alcohol-cirrosis, mala alimentación-diabetes, múltiples parejas-VIH/SIDA), sin considerar que detrás de ellas pueda haber factores estructurales como el contexto sociopolítico, el tipo de estado, el modelo de desarrollo, las políticas de acceso a educación y salud y otros.

Lipsky (2010) señala que los trabajadores de los servicios públicos tienden a etiquetar a los clientes como una reacción al exceso de trabajo, es decir, terminan delimitando quién es digno de recibir atención y quién no. Para ello utilizan el riesgo como una categoría discursiva que permite delimitar a los usuarios entre normales anormales y funcionales-disfuncionales y a partir de esto justifican su intervención con aquellos sujetos que se cree son "incapaces de ejercer su autonomía responsablemente, y que no pueden o no quieren administrar sus vidas o conducir sus riesgos" (Pollack, 2010). 
El inconveniente es que este tipo de categorizaciones pueden al mismo tiempo, transformarse en mecanismo de opresión y asignar tratamientos degradantes e inhumanos (Kemshall, 2010) en contra de los individuos que poseen ciertas características, como la clase social, el género, la orientación sexual, etc. Por ejemplo, Dominelli (1998) “habla de la 'cuádruple opresión' de la raza, el género, la clase social y la orientación sexual que afecta a las mujeres negras, lesbianas y de clase trabajadora". Por tanto, no es un contrasentido afirmar que nos movemos en un mundo donde lo que es calificado como riesgoso en realidad tiene una fuerte carga simbólica que puede limitar la oferta de oportunidades de quien esté bajo sus categorías.

Además, este tipo de conductas no tiene ningún efecto en la intervención que se realiza con los sujetos, puesto que está demostrado que "el énfasis en las necesidades humanas comunes en lugar de los problemas sociales mitiga el problema del proceso de etiquetamiento y ayuda a iluminar las numerosas vías que las personas utilizan para encontrar sus necesidades sin la necesidad de ser etiquetados como desviados o deficientes" (Kenneddy, 1995).

\section{El riesgo como peligro}

Bourdieu destaca que las categorías con las que pensamos y obramos no son sólo construcciones científicas, sino que además son componentes integrales de las estructuras y de lógicas sociales (Bourdieu, 1999). Por tanto, el riesgo en su calidad de categoría técnica también tendría una importante carga ideológica dependiendo del lugar desde el que se le esté observando.

Por ejemplo, desde la epidemiología el riesgo equivale al efecto, o probabilidad de ocurrencia de una patología en una población determinada, expresado a través del indicador paradigmático de incidencia (Filho, Castiel, \& Ayres, 2009). Por otra parte, desde la sociología de Niklas Luhman es la posibilidad de experimentar un posible daño como consecuencia de una decisión, de lo cual es imposible protegerse, porque toda decisión puede dar lugar a respuestas no deseadas (Filho et al., 2009).

Es así como estos dos discursos independientes de su origen "revelan una superposición semántica entre la idea del "peligro" y el "riesgo" (Filho et al., 2009), ya que, aunque Luhman realice una distinción entre ambos conceptos, este último siempre va a representar la "probabilidad del peligro, que generalmente se observará como una amenaza física para el hombre y /o para el medio ambiente" (Filho et al., 2009).

En este escenario, vale la pena preguntarse en qué nivel de preocupación se sitúa el riesgo. Lo que realmente nos importa es encontrar la verdadera razón de los resultados de negativos de salud o si necesitamos identificar a los responsables de sus circunstancias adversas.

Para la Organización Mundial de la Salud los factores de riesgo son "cualquier rasgo, característica o exposición de un individuo que aumente su probabilidad de sufrir una enfermedad o lesión" (OMS, 2013). Desde esta perspectiva pareciera ser que todo reviste peligro, pero claramente esto no es así, no es lo mismo ser mapuche, ser joven y vivir en un barrio calificado como peligroso, o tener malas compañías, ya que todo esto podría encarnar el espíritu lo que Foucault llama "sujeto bajo sospecha" de estar en riesgo.

Todos estos "riesgos" pueden ser entendidos a partir de esta idea del peligro, que finalmente puede hacer que tratemos estas cuestiones como naturales y fácticas y en el extremo de su uso, finalmente discriminemos todos eso que consideremos peligroso revestido de un dato técnico. 


\section{Nuevas perspectivas teóricas para abordar los problemas de salud}

Como hemos visto a lo largo de este documento el sistema de salud público está en deuda, porque tanto los "policymakers" como los implementadores hacen un uso indiscriminado del concepto de riesgo y no han sido capaces de girar hacia teorías contemporáneas que entreguen una forma distinta de abordar los complejos problemas de salud de las personas.

Teorías como el capital social, la perspectiva de las fuerzas y el empoderamiento nos darían la oportunidad de desarrollar nuevos modelos de intervención a través de la identificación, uso y aumento de las fuerzas de las personas y sus contextos, porque incluso en los contextos más adversos los sujetos, comunidades, ambientes, cuentan con recursos que pueden ser activados o movilizados para mejorar su calidad de vida (Arriagada Editora, 2015).

Serrano (Arriagada Editora, 2015), plantea que una intervención situada desde el capital social (Franke, Harnecker, Sanz, \& Pineda, 2008) permite mejorar el posicionamiento, las posibilidades y acceso a recursos de los actores, actúa como base social y cultural de los intercambios económicos, facilita que las personas comprendan proyectos en común, sobre la base de normas compartidas y expectativas respecto del comportamiento del otro y favorece las virtudes cívicas. Ejemplo de esto es lo ocurrido en Kerala, estado de la India, dónde un grupo de políticos, economistas y expertos decidió sustituir la limitada idea de desarrollo económico por la noción de capital social a la hora de intervenir y los resultados han sido verdaderamente asombrosos. En este territorio una quinta parte de la población subsiste con menos de un dólar al día, más de un tercio son analfabetos, mientras que más de cien millones no tienen acceso a las mínimas atenciones médicas, ni a educación. Sin embargo, paradojalmente la expectativa de vida es de 72 años (cifra muy cercana a la media americana de 76 años), la mortalidad infantil es una de las más bajas de los países en vías de desarrollo de todo el mundo, el crecimiento demográfico está bajo control, porque el índice de fertilidad es de 1.7 nacimientos por mujer, más bajo que en Suecia o en América. Todo lo que en su conjunto desafía la clásica concepción de que el ingreso económico es un factor de riesgo que limita la calidad de vida.

Por otra parte, intervenir desde la perspectiva de las fuerzas, permite centrarse en los recursos de las personas, disminuir la perspectiva de inevitabilidad de las deficiencias y mejorar las competencias de los sujetos. Por ejemplo, un estudio evaluó a 632 personas que tenían problemas con el abuso de sustancias y encontraron que aquellos que habían estado sometidos a una intervención desde este enfoque tenían menos probabilidades de recaer que aquellos que eran sometidos a intervenciones tradicionales (Siegal et al., 1996).

$\mathrm{Al}$ igual que las perspectivas anteriores, el empoderamiento es una teoría que nace en Estados Unidos enmarcada en una visión ecológica en reacción a intervenciones estandarizadas, que no consideraban los contextos sociales y experiencias de los individuos (Rappaport \& Seideman, 2012). Este enfoque buscar que las personas, familias, comunidades, se reapropien y adquieren poder y dominio sobre sus vidas y además busca transformar las relaciones sociales opresivas y dominantes que impiden la participación real de las minorías socioculturales en los procesos de toma de decisión (Rappaport \& Seideman, 2012). Para ello, es menester reconocer que el poder es inherente a todas las profesionales de la salud que trabajan directamente con personas y que en esa relación subyace inevitablemente la desigualdad. Por tanto, tal vez no es posible distanciarse de ella, pero si reconocerla, con el fin abandonar las prácticas opresivas que limitan las oportunidades de los grupos más desfavorecidos.

Todas estas perspectivas se caracterizan porque colocan la centralidad de la atención en "los recursos de las personas y sus medios ambientes, más que sus patologías y problemas" (Saleebey, 1996), a diferencia del enfoque de riesgo que a menudo culpa a las víctimas. Girar hacia estas corrientes teóricas es muy importante, porque si se continúa observando a los sujetos desde la perspectiva del riesgo, se seguirán utilizando modelos convencionales de intervención que no logran generar una real oferta de oportunidades. 
Además, el problema no es la falta de demanda por parte de los usuarios, es la falta de oferta. Por años nos hemos focalizado en la definición de los riesgos y las incapacidades de los sujetos sin hacernos cargo del hecho de que todos los hombres son actores conscientes de su propio desarrollo. En este sentido las perspectivas mencionadas, a diferencia del enfoque de riesgo, colocan un especial énfasis en la autonomía de las personas, es decir, en palabras de Kant, en su capacidad de auto legislarse.

No obstante, todo esto, necesariamente implica dejar atrás el uso indiscriminado del término riesgo y "creer que las personas son capaces de tomar sus propias elecciones y decisiones, asumir que el ser humano posee la fuerza y el potencial para resolver sus propias situaciones difíciles, reconocer que los sujetos son capaces de aumentar sus fuerzas" (Saleebey, 1996).

\section{Conclusión}

Cuando el sistema de salud se orienta a intervenir exclusivamente desde la perspectiva del riesgo, parte de un punto cero, desde el déficit, es decir se olvida de los recursos y fortalezas con que cuentan las personas. A mi juicio esto tiene una serie de implicancias: en primer lugar impide que los profesionales de la salud comprendan los problemas integralmente, porque siempre hay recursos con los que se puede intervenir y en segundo lugar, esto podría acarrear efectos negativos en la identificación de los problemas que enfrentan quienes experimentan necesidades complejas, como objeto de intervención, en tanto, el modelo de detección del riesgo promovido por el sistema de salud, podría transformase en un mecanismo de opresión.

El "riesgo" es un concepto sociológico e ideológico, pero no genético, no es constitutivo de las personas, solo es el resultado de las condiciones de vida más extremas, del déficit de la sociedad en su conjunto y de las ofertas que el sistema de salud ofrece. Que los habitantes de Vitacura tengan en promedio una esperanza de vida de 83 años y que en San Miguel esta sea de 74 años, habla de lo que hemos creado, de la segregación territorial, de las diferencias socioeconómicas, de las políticas mal diseñadas y de los diferentes sistemas de salud a los que estas personas pueden acceder.

Los indicadores de riesgo han favorecido una adhesión simplista a las hipótesis. El problema de esto es que tal como lo plantea Adela Cortina, estamos en un mundo esquizofrénico, en que hay profundas inequidades, por lo tanto, es necesario ir en pos de la comprensión de esa complejidad para poder reducirla y eso necesariamente implica dejar de observar la realidad exclusivamente desde el riesgo y girar hacia otras perspectivas teóricas.

Muchas personas nacen con un grupo de factores de riesgo acumulados sin esquemas explicativos, que se presentan como una colección infinita de factores que, en última instancia, explican muy poco los orígenes de las enfermedades. No obstante, dejar de utilizar indiscriminadamente el riesgo y mirar otras perspectivas, nos permiten dejar de responsabilizar a los sujetos de sus malas condiciones de salud y mirar la real oferta de oportunidades de los sistemas sanitarios.

Las categorías discursivas asociadas al riesgo "son estructuras de conocimientos, afirmaciones y prácticas mediante las cuales comprendemos, explicamos y decidimos cosas... Son marcos o cuadrículas de organización social que hacen posibles ciertas acciones e impiden otras" (Healy, 2001), por consiguiente, no es lo mismo intervenir con personas que son observados desde el déficit (discapacitados, prostitutas, pacientes, etc.) que con sujetos con recursos (personas con necesidades especiales, trabajadoras sexuales, ciudadanos, etc.).

En este escenario el capital social, el empoderamiento y la perspectiva de las fuerzas ofrecen la posibilidad de articular los diferentes problemas que las personas pueden experimentar y superar los dualismos simplistas mediante el uso de sus recursos. Las fuerzas permitirían pensar en las paradojas de la modernización y traducirlas en acciones que enfrenten la inequidad en salud, ya que este tipo de análisis permite dejar de ver los indicadores de riesgo como cuestiones naturales y fácticas. 
Además, el enfoque de riesgo contribuye a generar una lógica de desconfianza generalizada hacia aquellos usuarios que son considerados peligrosos, pero esto también repercute negativamente en los equipos de trabajo del sistema de salud que creen que nunca van a lograr sus objetivos. Por tanto, esto se puede transformarse en un extraordinario malgasto de recursos, sin ningún espacio para colocar cuestiones importantes.

Para finalizar, es necesario señalar que cambiar las perspectivas teóricas con que se abordan las necesidades de los beneficiarios del sistema de salud, podría generar una real oferta de oportunidades. Desde luego no un 100 $\%$ de éxito, es imposible pensar en hospitales sin pacientes, o en un $100 \%$ de equidad. No obstante, hay que hacer un esfuerzo por avanzar hacia la construcción de un sistema que incluya los riesgos y los recursos, con la firme convicción de que eso importan, porque las personas importan, porque pensar y obrar esta manera abriría oportunidades a sujetos que podrían ser extraordinariamente talentosos.

\section{Bibliografía}

1. Arellano, R. M. (2010). La "medida" de lo posible Cuantificación y esfera pública en Chile. Viña del Mar.

2. Arriagada Editora, I. (2015). Aprender de la experiencia El capital social en la superación de la pobreza Comisión Económica para América Latina y el Caribe. Santiago.

3. Beddoe, L. (2010). Surveillance or Reflection: Professional Supervision in "the Risk Society." British Journal of Social Work, 40(4), 1279-1296. https://oi. org/10.1093/bjsw/bcq018

4. Bourdieu, P. (1999). La Miseria del Mundo. Madrid: Akal.

5. Dominelli, L. (1998). Anti-oppressive practice in context. Social Work, 3-22.

https://doi.org/10.1007/978-1-349-14400-6_1

6. El Ciudadano. (2020, March). Defensora de la Niñez llama a denunciar a Radio Agricultura por "falacias" de carácter sexual que aluden a menores de edad. Retrieved from https://www.elciudadano.com/chile/defensora-de-la-ninez-llama-a-denunciar-a-radio-agricultura-por-falacias-de-caracter-sexual-que-aluden-a-menores-deedad/03/06/

7. El Mercurio. (2019, January 31). Las personas casadas tienen menos riesgo de infarto.

8. Filho, N. D. A., Castiel, L. D., \& Ayres, J. R. (2009). Riesgo: - básico de la epidemiología. Salud Colectiva, 5(3), 323-344 https://doi.org/10.18294/sc.2009.238

9. Franke, R., Harnecker, M., Sanz, A., \& Pineda, C. (2008). Estado Kerala, India: Una experiencia de planificación participativa descentralizada (Centro Internacional Miranda, Ed.). Retrieved from https://www.researchgate.net/publication/303287056_ESTADO_KERALA_INDIA_UNA_EXPERIENCIA_DE_PLANIFICACION_ PARTICIPATIVA_DESCENTRALIZADA

10. Healy, K. (2001). Trabajo Social: Perspectivas Contemporáneas (1st ed.). Madrid: Ediciones Morata

11. Kemshall, H. (2010). Risk Rationalities in Contemporary Social Work Policy and PracticeNo Title. The British Journal Social Work, 40(4), 1247-1262. Retrieved from https://academic.oup.com/bjsw/article-abstract/40/4/1247/1619111

12. Kenneddy, R. (1995). Social Policy Development: The Strengths Perspective. KENNEDDY CHAPIN, Rosemary, 40(4), 501-514

13. Krieger, N. (2002). Glosario de epidemiología social. Revista Panamericana de Salud Pública, 11(5-6), 480-490.

https://doi.org/10.1590/s1020-49892002000500028

14. Lipsky, M. (2010). Street-Level Bureaucracy, Dilemmas of the Individual in Public Service (30th ed.). New York: The Roussel Sage Foundation.

15. Mercurio, E. (2020). Minsal detalla seguimiento de casos "en riesgo" de coronavirus: se les recomienda evitar lugares concurridos $\mid$ Emol.com.

16. Montenegro, S. M. (2005). La Sociología de la Sociedad del Riesgo: Ulrich Beck y sus Críticos. Pampa, (1), 117-130. https://doi.org/10.14409/pampa.v1i1.3122

17. Mostrador, E. (2014). Miguel Otero asegura que hijos fuera del matrimonio tienen coeficiente intelectual menor que los de "una familia de padre y madre normal" - El Mostrador. Retrieved from https://www.elmostrador.cl/noticias/pais/2014/01/08/miguel-otero-asegura-que-hijos-fuera-del-matrimonio-tienen-coeficiente-intelectualmenor-que-los-de-una-familia-de-padre-y-madre-normal/

18. OMS. (2013). OMS | Una nueva publicación de la OMS analiza los factores de riesgo para la salud.

19. Pollack, S. (2010). Labelling clients 'risky': social work and the neo-liberal welfare state - Social Care Online. British Journal Social Work, $40(4)$, $1263-1278$. Retrieved from https://www.scie-socialcareonline.org.uk/labelling-clients-risky-social-work-and-the-neo-liberal-welfare-state/r/a1CG0000000GZheMAG

20. Programa de Naciones Unidas para el Desarrollo. (2009). La Manera de Hacer las Cosas. Retrieved from http://desarrollohumano.cl/idh/download/pnud_2009.pdf 21. Rappaport, J., \& Seideman, E. (2012). Handbook of Community Psychology - Google Libros. Retrieved from https://books.google.cl/ books?id=ypoyBwAAQBAJ\&dq=RAPPAPORT,+J.,+SEIDMAN,+E.\&hl=es\&lr=

22. Saleebey, D. (1996). The Strengths Perspective in Social Work Practice: Extensions and Cautions. Social Work, 41(3), 296-305. https://doi.org/10.1093/sw/41.3.296

23. Siegal, H. A., Fisher, J. H., Rapp, R. C., Kelliher, C. W., Wagner, J. H., O’Brien, W. F., \& Cole, P. A. (1996). Enhancing substance abuse treatment with case management: Its impact on employment. Journal of Substance Abuse Treatment, 13(2), 93-98. https://doi.org/10.1016/0740-5472(96)00029-3

24. Tercera, L. (2018, July 27). Experiencias a lo largo de la vida influyen en el envejecimiento cerebral - La Tercera. Retrieved from https://www.latercera.com/noticia/ experiencias-a-lo-largo-de-la-vida-influyen-en-el-envejecimiento-cerebral/ 